\title{
Selected Problems of Optimization of a Switched Reluctance Motor for an Electric Vehicle using Analytical Calculations
}

\author{
M. Majchrowicz ${ }^{1}$, W. Jazdzynski ${ }^{1}$ \\ ${ }^{1}$ Elec. Eng., Automatics, Comp. Sc. and Electronics Dept., AGH University of Science and Technology \\ Al. Mickiewicza 30, 30-059 Cracow (Poland)
}

Phone: (+48)-(12)-617-28-98, Fax number: (+48)-(12)-634-10-96, e-mail: m.majchrowicz@yahoo.pl, wjaz@agh.edu.pl

\begin{abstract}
A model and an example of optimization of a $6 / 4$ type SRM, designed for an electric vehicle, is described in the paper. The modelling procedure, based on simplified analytical calculations, allows for estimation of the torque, efficiency and acoustic noise of the motor, taking into account the magnetic non-linearity. Some problems arising from the existence of mutual inductances are described as well. Calculations have been performed in MATLAB environment, and verified by means of FEM analysis.
\end{abstract}

\section{Key words}

Switched reluctance motor (SRM), electric vehicle, optimization, analytical model.

\section{Introduction}

There are several drawbacks when applying switched reluctance motors - torque ripples, small efficiency at high velocity and acoustic noise, which decide about its competitiveness in comparison with other types of electric motors (i.e. induction motor), especially in electric car drive applications. The great advantage is a simple construction and a high reliability. The paper describes a procedure to develop a 6/4 type SRM with the best performances. Some information related to this construction presented in an earlier paper [1] has been taken into account.

A gradient optimization procedure has been employed to improve performances of the SRM. The procedure uses a modified version of the analytical model of the SRM described in [2, 3]. The improved model enables estimation in the steady state operation of not only efficiency and torque ripples, but also acoustic noise, and furthermore - takes into account the magnetic nonlinearity. The model does not take into account mutual inductances and their influence on optimization results. A simplified solution of the problem has been proposed in the paper. Calculations were performed in MATLAB environment. The solutions were verified by means of FEM analysis.
The analytical model of the SRM is described in the first part of the paper. The problem of neglecting mutual inductances in modelling is discussed. Some results of an optimization approach are presented as well.

\section{Analytical Model}

The analytical model allows for estimation of all important motor quantities, for instance the phase current, electromagnetic torque, flux density in the stator pole, and radial force operating on stator pole of the SRM. There is assumed a constant velocity and one pulse mode operation of the motor. The input variables are geometrical dimensions (Fig. 1) and such parameters as supplying phase voltage, input active power (or angle $\varphi_{\text {off }}$ defining a moment of switching off the voltage), rotor angular velocity, phase winding turn number, and a diameter of a conductor.

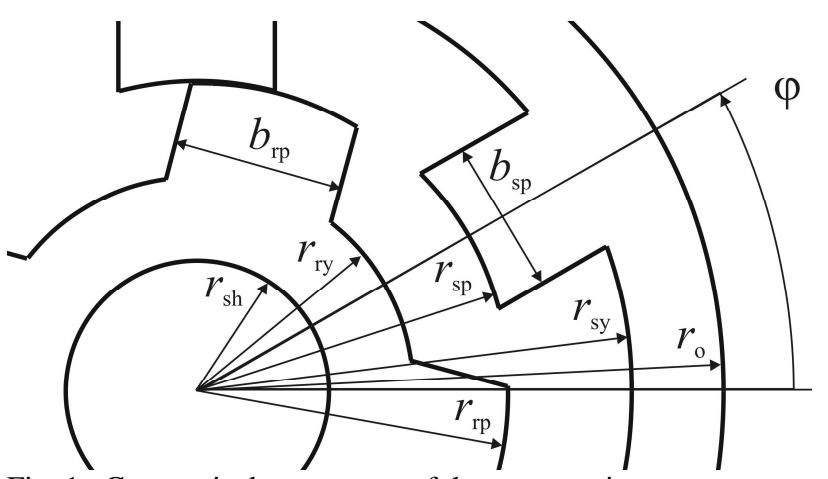

Fig. 1. Geometrical parameters of the construction.

The inductance $L$ of a phase winding is calculated at the very beginning, applying the reluctance network method, as a function of phase current and angular rotor position. A procedure of calculating the inductance has been described in $[4,5]$. The cross-section area of the core is divided on six regions, each with a flux assumed to be constant (called "flux tubes", Fig. 2). Every flux tube represents a magnetic circuit, where its flux value is a solution of a non-linear equation. The inductance of the phase winding is a sum of inductances of all tubes. 

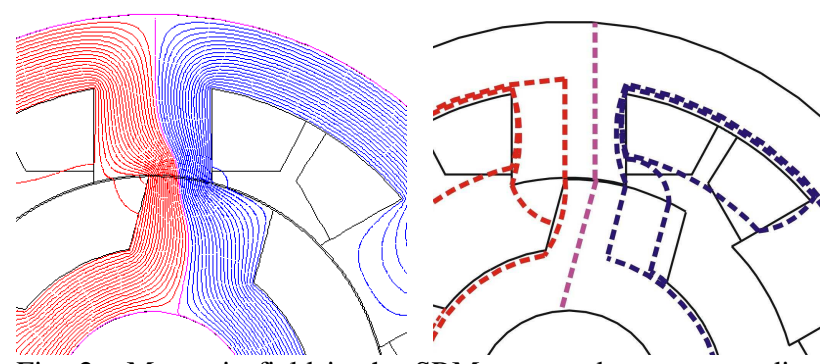

Fig. 2. Magnetic field in the SRM core and a corresponding flux tubes arrangement, for a selected rotor position.

Next a function of a flux linkage and its both partial derivatives (with respect to phase current and angular rotor position) are calculated.

A reason for such a calculation order is an earlier research work devoted optimization [2, 5], focused on some substituted criterion functions arising from the inductance of a phase winding.

The torque function is calculated from a magnetic coenergy function, as a function of the angle $\varphi$, for several constant values of the $i_{\mathrm{x}}$ :

$$
T_{e l}\left(i_{\mathrm{x}}, \varphi\right)=\frac{\partial}{\partial \varphi} \int_{0}^{i_{\mathrm{x}}} L(i, \varphi) i \mathrm{~d} i
$$

The next step is a calculation of the phase winding resistance, which depends on dimensions of the stator pole, as well as winding parameters, all influenced by the optimization procedure. It comprises an additional resistance of the converter elements, the constant voltage source and all wires as well.

Obtained resistance and partial derivatives of the flux linkage $\Psi(i, \varphi)$ are used to obtain the function of a phase current $i(t)$ as an analytical solution of the following nonlinear differential equation:

$$
u=R i+\frac{\partial \Psi(i, \varphi)}{\partial i} \frac{\mathrm{d} i}{\mathrm{~d} t}+\omega \frac{\partial \Psi(i, \varphi)}{\partial \varphi}
$$

where:

$$
\begin{array}{ll}
u & - \text { phase voltage } \\
i & - \text { phase current } \\
t & - \text { time } \\
\omega & - \text { angular velocity } \\
\varphi & - \text { angular rotor position } \\
\Psi & \text { - flux linkage } \\
R & \text { - phase resistance }
\end{array}
$$

The equation is solved for a rotor angular velocity $\omega=n^{*} \pi / 30$ assumed to be constant, for $n=3500 \mathrm{rpm}$, in an angle interval $\left[0,2 \pi / N_{\mathrm{r}}\right]$ (between two consecutive unaligned stator-rotor positions; $N_{\mathrm{r}}$ is a number of rotor poles), for one phase winding. The equation is solved for one phase, under conditions:

$$
\begin{aligned}
& u(0)=E ; \quad i(0)=0 ; \quad i \geq 0 \\
& u= \begin{cases}E, & \text { if } \quad 0 \leq \varphi \leq \varphi_{\text {off }} \\
-E, & \text { if } \quad \frac{2 \pi}{N_{\mathrm{r}}} \geq \varphi>\varphi_{\text {off }} .\end{cases}
\end{aligned}
$$

where $E$ is the voltage value of the constant voltage source assumed in the paper to be $160 \mathrm{~V}$. The phase current is assumed to be not lower than zero, because of an assumed inverter configuration. The quantity $\varphi_{\text {off }}$ is its turn-off angle, value of which is selected through a solution of a proper non-linear algebraic equation in each optimization iteration, to obtain the reference value $35 \mathrm{~kW}$ of the motor input active power. Both poles of one phase are connected in parallel. These assumptions are valid in all calculations performed in this paper.

The non-linear differential equation (2) has been solved analytically because of the troubles with a convergence of the optimization routine appeared in [2]. Fortunately, the flux derivatives can be represented by its piece-wise constant approximation with a good accuracy (in the model they are calculated for all $k$ using look-up tables). If in an $k$ th time interval $\left[t_{0}{ }^{k}, t_{\mathrm{e}}{ }^{k}\right]$ holds:

$$
\frac{\partial \Psi(i, \varphi)}{\partial i}^{k}=\text { const }_{i k}, \frac{\partial \Psi(i, \varphi)}{\partial \varphi}^{k}=\text { const }_{\varphi k},
$$

than an analytical solution exists:

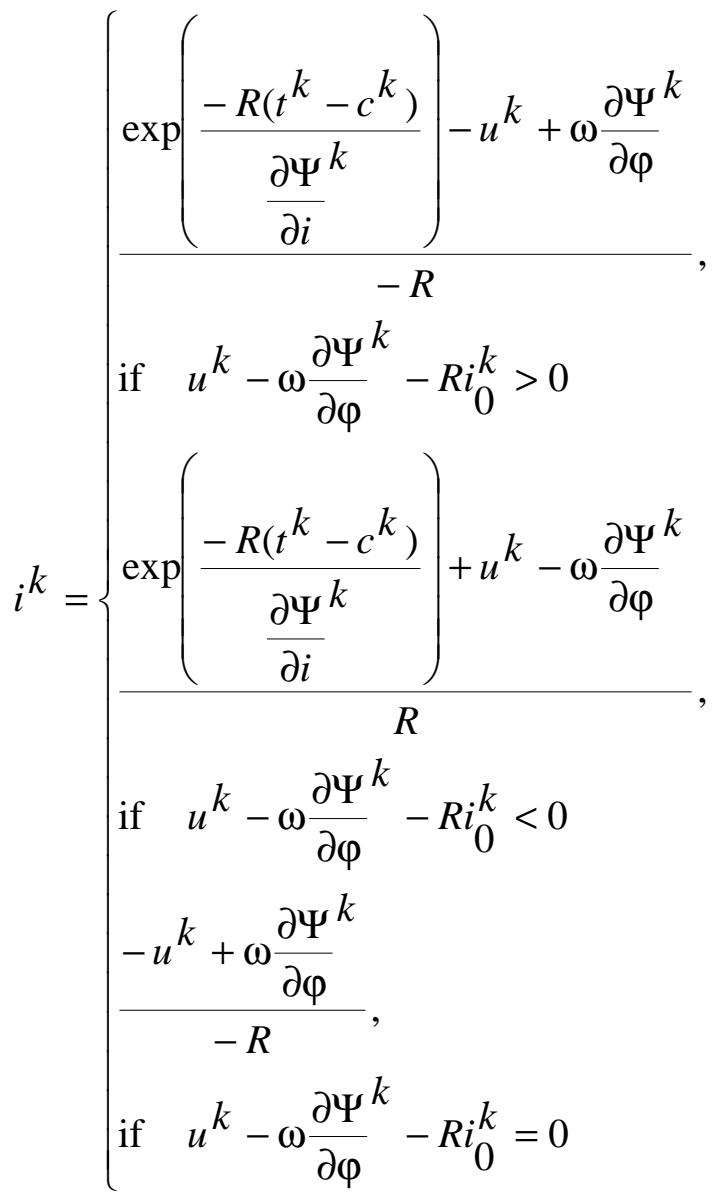


where $c^{k}$ is constant and results from initial conditions. In the next step the following quantities are calculated (using look-up tables): time functions of a torque, a flux density in a stator pole and a radial force. The radial force is calculated according to equation:

$$
F_{\mathrm{rad}}^{\mathrm{k}}=L_{4 \mathrm{Frad}}\left(i^{k}, \varphi^{k}\right) \cdot\left|-\frac{\left(\phi_{\text {aligned }}\left(i^{k}, \varphi=\frac{\pi}{4}\right)\right)^{2}}{2 \mu_{0} l\left(r_{\mathrm{rp}} \frac{\beta_{\mathrm{s}}+\beta_{\mathrm{r}}}{2}+g\right)}\right|
$$

where $L_{4 \mathrm{Frad}}$ is an auxiliary function (of the angle $\varphi$ and phase current $i$ ) which depends on the shape of functions $L(\varphi, i=\mathrm{const})$ and $F_{\mathrm{rad}}(\varphi, i=\mathrm{const}), \beta_{\mathrm{s}}$ and $\beta_{\mathrm{r}}$ are the stator and rotor pole arcs, $g$ is the air-gap length, $l-$ is the stack length, the radius $r_{\text {rp }}$ is defined on Fig. $1, \mu_{0}$ is magnetic permeability of a vacuum and $\phi_{\text {aligned }}$ is a magnetic flux at an aligned position of the rotor.

\section{Criterial Functions in Optimization}

The model allows for estimation of the most important criterial functions in optimization, it means: an average value of the total electrical torque $T_{\mathrm{av}}$ [2], a ratio of an average value of the electrical torque to its maximum value (the torque ripples quantity) $T_{\mathrm{av} 2 \mathrm{~m}}$ [2], the efficiency of the motor, an acoustic noise level $L_{\mathrm{Aw}}$, and a total mass $m$ (or cost of the materials).

Efficiency is defined in the same way as in [2], it means:

$$
\eta=\frac{P_{\text {in }}-\Delta P_{\mathrm{Fe}}-\Delta P_{\mathrm{Cu}}-\Delta P_{\text {mech }}}{P_{\text {in }}} \cdot 100 \%
$$

where the power components are defined as follows:

$P_{\text {in }} \quad-$ input active power (8)

$\Delta P_{\mathrm{Cu}} \quad$ - electrical loss in the stator winding (9)

$\Delta P_{\mathrm{Fe}} \quad$ - iron loss in the magnetic core $(10,11,12)$

$\Delta P_{\text {mech }}-$ mechanical power loss as in $[2,7]$

$$
\begin{gathered}
P_{\text {in }}=\frac{\omega}{2 \pi} N_{\mathrm{r}} N_{\mathrm{ph}} \sum_{k} \int_{t_{0}^{k}}^{t_{\mathrm{e}}^{k}} u^{k} k^{k} \mathrm{~d} t \\
\Delta P_{\mathrm{Cu}}=\frac{\omega}{2 \pi} N_{\mathrm{r}} \frac{N_{\mathrm{s}}}{2} \frac{R_{\mathrm{pole}}}{2} \int_{0}^{\frac{1}{N_{\mathrm{r}}} \frac{2 \pi}{\omega}} i^{2} \mathrm{~d} t
\end{gathered}
$$

The $N_{\mathrm{s}}$ is a number of stator poles, $N_{\mathrm{ph}}$ is a number of phases, and $R_{\text {pole }}$ is a resistance of one pole winding (the inverter loss was omitted in the analysis).

An accurate determining of the iron loss is troublesome due to a complex shape of the flux density functions in different machine parts [2, 7]. The iron losses are calculated in a simplified way, based upon the assumption that the functions of magnetic flux density can be approximated by means of a linear spline. An iron loss formula applied in the paper comprises hysteresis $\Delta P_{\text {Fehx }}$ and eddy-current $\Delta P_{\text {Feex }}$ components:

$$
{ }^{\Delta P_{\mathrm{Fe}}}=\Delta P_{\text {Fehx }}+\Delta P_{\text {Feex }}
$$

An example of the calculation procedure related to the iron loss in the rotor poles is described below. If the flux density function in the rotor pole is as on Fig. 3,

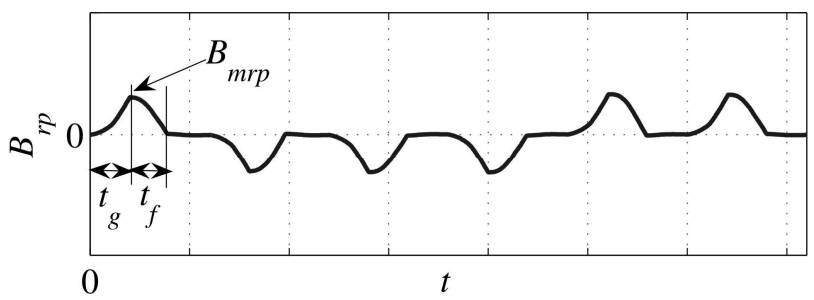

Fig. 3. Flux density function in the rotor pole for one complete rotation of the rotor (FEM calculations for $n=3500 \mathrm{rpm}$ ).

than:

$$
{ }^{\Delta P_{\text {Fehrp }}}=C_{\mathrm{h}}\left(\left(N_{\mathrm{s}}-4\right) \cdot 0.4+2\right) \frac{\omega}{2 \pi} B_{\mathrm{mrp}}^{2} N_{\mathrm{r}} V_{\mathrm{rp}} k_{\mathrm{stk}}
$$

and

$$
\begin{aligned}
& \Delta P_{\text {Feerp }}=C_{\mathrm{e}}\left[\left(\frac{B_{\mathrm{mrp}}}{t_{\mathrm{g}}}\right)^{2} t_{\mathrm{g}}+\left(\frac{B_{\mathrm{mrp}}}{t_{\mathrm{f}}}\right)^{2} t_{\mathrm{f}}\right] \\
& \cdot N_{\mathrm{s}} \frac{\omega}{2 \pi} N_{\mathrm{r}} V_{\mathrm{rp}} k_{\mathrm{stk}}
\end{aligned}
$$

where $V_{\mathrm{rp}}$ is a volume of one rotor pole, $C_{\mathrm{h}}$ and $C_{\mathrm{e}}$ are the coefficients of hysteresis and eddy-current losses. The total iron loss function can be obtained after applying a similar procedure to the other parts of the machine, basing upon corresponding flux density functions presented on Figures 5 and 6 in [2]. The model of iron losses is true on the assumption that the $\varphi_{\text {off }} \leq 30^{\circ}$.

Acoustic noise calculations are based on a simplified analytical model described in $[6,7,8]$. The elements of the noise calculations are: a radial force time function, a modal analysis of the stator, a frequency domain analysis of the radial force, an amplitude of dynamic deflection of the stator, and a sound power radiated by a motor.

The radial force function in SRM is a non-sinusoidal waveform, so the acoustic noise analysis has to be done for every important sinusoidal component of the radial force function. Furthermore, the sound power level of every frequency component has to be weighted (Aweighting curve), because of the human ear properties. The A-weighted sound power levels $L_{\mathrm{wA} h}$ of each frequency component are combined to give one single 
value $L_{\mathrm{wA}}$ to compare different constructions of the motor (13).

$$
L_{\mathrm{wA}}=10 \log \left(\sum_{h} 10^{\left.0.1 L_{\mathrm{wA} h}\right)}\right)
$$

If the noise level is analysed on the area of the source of radiation, the sound power level of a single frequency component can be expressed as:

$$
L_{\mathrm{w}}\left(f_{\text {exc }}\right)=10 \log \left(\frac{P_{\text {sound }}\left(f_{\text {exc }}\right)}{P_{\text {sound, ref }}}\right)
$$

where $P_{\text {sound }}$ is the sound power radiated by the motor and $P_{\text {sound,ref }}$ is a reference sound power equal $10^{-12} \mathrm{~W}$.

The sound power $P_{\text {sound }}$ depends on excitation frequency $f_{\text {exc }}$ as follows:

$$
P_{\text {sound }}\left(f_{\text {exc }}\right)=\sum_{m} 4 \sigma_{\text {rel }} \rho_{\text {air }} c_{\text {air }} \pi^{3} f_{\text {exc }}^{2} D_{\text {circum }}^{2} r_{\mathrm{o}} l
$$

where:

$$
\begin{array}{ll}
m & - \text { order of a mode } \\
\sigma_{\text {rel }} & - \text { modal radiation efficiency [8] } \\
c_{\text {air }} & - \text { travelling speed of sound in the air } \\
\rho_{\text {air }} & - \text { density of air } \\
D_{\text {circum }} & - \text { amplitude of dynamic deflection (16) }
\end{array}
$$

The formula for amplitude of dynamic deflection is [8]:

$$
\begin{aligned}
& D_{\text {circum }}\left(f_{\text {exc }}\right)=\frac{F_{\text {rad }, h}}{M_{\mathrm{s}} 4 \pi^{2} f_{m}^{2}} \cdot \\
& \cdot \frac{1}{\left[1-\left(\frac{f_{\text {exc }}}{f_{m}}\right)^{2}\right]^{2}+\left[2 \zeta_{m}\left(\frac{f_{\text {exc }}}{f_{m}}\right)\right]^{2}}
\end{aligned}
$$

where:

$F_{\text {rad }, h} \quad-h$-order frequency component of the radial force

$f_{m} \quad$ - frequency of mode $m$ calculated in a simplified way similar to $[6,7,8]$

$M_{\mathrm{s}} \quad$ - mass of the stator system

$\zeta_{\mathrm{m}} \quad-$ damping factor $[8]$

The modal analysis was done for the modes $m=0,2,4$.

\section{Validation of the Analytical Model by Means of FEM Calculations; Problem of Mutual Inductances Existence}

The quality of the optimization results depends strongly on an accuracy of the applied model. The validation calculations concerning the analytical model were performed by means of a $2 \mathrm{D}$ finite element analysis.
Phase current functions obtained for both models are shown on Fig. 4.

Simulation calculations have been performed under conditions described in the second paragraph, but for an imposed turn-off angle $\varphi_{\text {off }}$, which does not arise from the input power requirement. The FEM analysis was performed for two cases - for only one phase supplied (like in the analytical model, without the mutual inductances, as Case I), and for all three phases supplied, to show the effect of including mutual inductance (Case II). The next phase has been switched on at $\varphi=30$ degrees of the rotor angular position. This value is a characteristic quantity for the analyzed motor construction type, equal to $\left(360^{\circ} / N_{\mathrm{ph}}\right) / N_{\mathrm{r}}$. Usually the mutual inductance has a small value in a comparison to the main inductance one. In non-linear systems the main inductance value depends on saturation, and sometimes can be of the same order as the mutual inductance. In the analysed motor the influence of the mutual inductance is remarkable when the current values in two different phase windings are high enough to saturate the core (i.e. when the first phase is switched off at $\varphi=35$ degrees, instead of 30 degrees as in Case I, and the current in the next phase is about 200A). Neglecting the mutual inductance in the model can generate some errors, see Fig. 4.
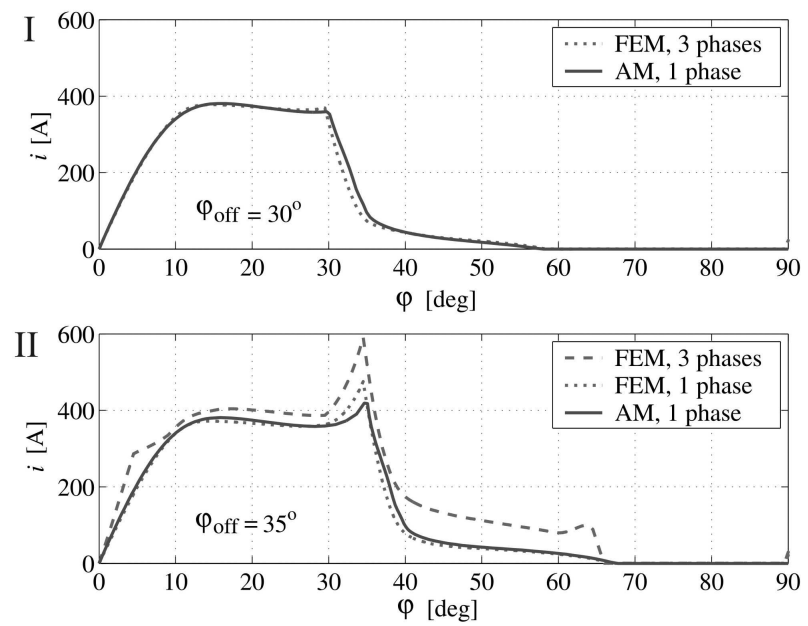

Fig. 4. Phase current function - a comparison of analytical (AM) and finite element models (FEM), for two values of the $\varphi_{\text {off }}$, and two cases of FEM simulations - for all three phases, and for one phase.

Some validation results are shown on Fig. 5 in the next paragraph.

\section{Optimization calculations}

A few examples of scalar optimization calculations have been performed to test the analytical model. Geometrical parameters defined on Fig. 1 (with the exception of the rotor pole radius $r_{\text {rp }}$ ), air-gap length, stack length and parameters of the winding have been assumed to be the 11 optimization variables. The feasible region $\mathrm{X}_{0}$ was defined by means of 33 linear and 15 non-linear inequality, and 1 non-linear equality constraints, which were chosen to prevent obtaining unrealistic optimization results, and to meet imposed design requirements. The 
equality constraint concerns the input power, and has been defined as: $P_{\text {in }}=35 \mathrm{~kW}$.

First of all an experiment has been performed to check the influence of neglecting the mutual inductance in the model on optimisation results. For angles $\varphi_{\text {off }}>30^{\circ}$ the effect of mutual inductance should be more visible. To show the effect, a scalar optimization problem (17) as in [3] has been solved three times, for three maximum values of the $\varphi_{\text {offmax }}: 30^{\circ}, 32^{\circ}$ and $35^{\circ}$.

$$
\begin{gathered}
\max _{\mathbf{x}} T_{a v 2 \max } \mid \mathbf{x} \in \mathrm{X}_{0}, T_{a v} \geq T_{a v 0}, \quad m \leq m_{0}, \\
\varphi_{\text {off }} \leq \varphi_{\text {offmax }},
\end{gathered}
$$

In the formula (17) the quantity $\mathbf{x}$ is a vector of optimization variables, $\mathrm{X}_{0}$ is the basic feasible region, $T_{\mathrm{av}}$ is the average torque, $T_{\mathrm{av} 2 \max }$ is the relation of the average value to the maximum value, and the $m$ is a total mass of the motor. The index " 0 " marks the initial construction described in [1]. Results of the experiments are shown on Fig. 5.
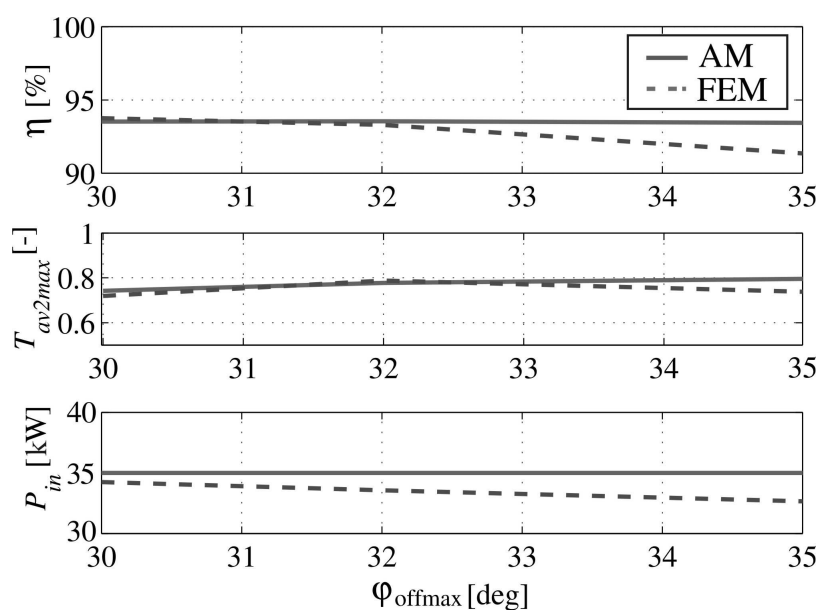

Fig. 5. The influence of neglecting the mutual inductance in the analytical model on optimization results - a comparison of analytical model (AM) - without the mutual inductance effect, and finite element model (FEM) - with the mutual inductance effect), for the constructions optimized with few values of the $\varphi_{\text {offmax }}$.

The results show that increasing the maximal feasible value of the angle $\varphi_{\text {off }}$ above the value about $30^{\circ}$ in optimization leads to obtain lowered values of the input power, efficiency and the $T_{\text {av2max }}$.

Finally, two tasks of searching for the optimal construction have been solved taking into account the result of the above considerations, it means a constraint on the maximum value of the $\varphi_{\text {off }}$ angle was imposed, $\varphi_{\text {off }} \leq \varphi_{\text {offmax }}=30^{\circ}$.

The goal of the first task, defined as a problem (17), was minimal value of the torque ripples. The electromagnetic torque functions obtained by means of the analytical and FEM models, for the initial construction and the optimal one, are presented on Fig. 6. Selected results of the optimization are presented on Fig. 7.

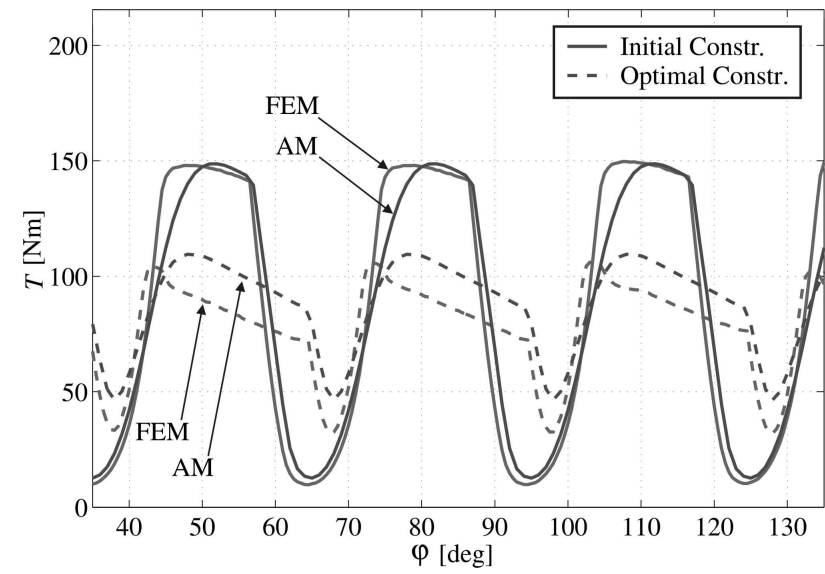

Fig. 6. Functions of the electromagnetic torque obtained with the help of an analytical model (AM), and finite element simulations (FEM), for the initial construction and the optimal one - minimization of the torque ripples (17).
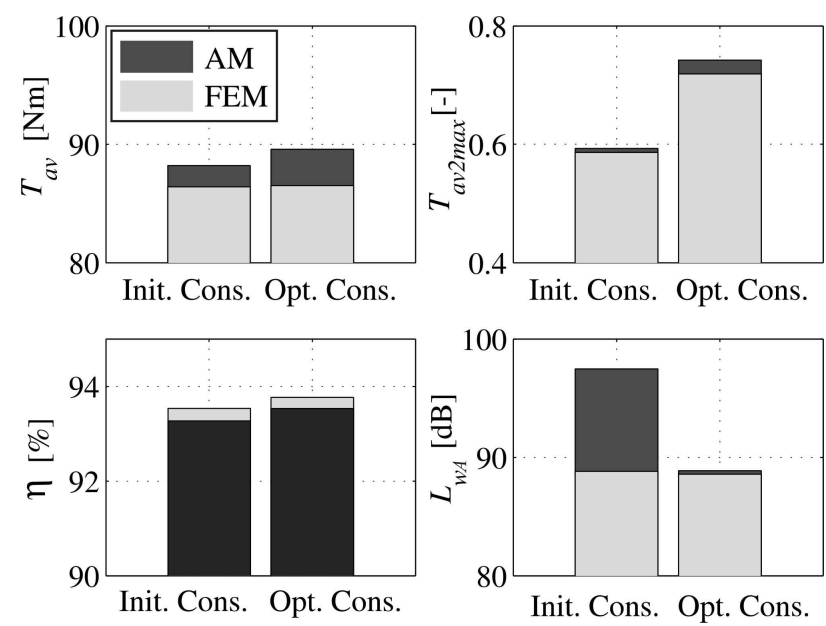

Fig. 7. Values of important motor quantities obtained with the help of an analytical model (AM), and finite element simulations (FEM), for the initial construction and the optimal one - minimization of the torque ripples, problem (17).

Both values of acoustic noise level, for AM (analytical model) and FEM, (Fig. 7 and 8) have been obtained with the help of the same analytical calculations (13)-(16), but for two different methods of the $F_{\text {rad }}$ function estimation. The difference between values $L_{\mathrm{wA}}$ for initial construction on Figures 7 and 8 is caused by high sensitivity of the analytical acoustic noise model on spectral differences between $F_{\text {rad }}$ functions obtained with the help of different methods.

The goal of the second task was maximal value of the motor efficiency (18). Selected results of optimization calculations are presented on Fig. 8.

$$
\begin{aligned}
& \underset{\mathbf{x}}{\max } \eta \mid \mathbf{x} \in \mathrm{X}_{0}, \ldots \\
& \ldots T_{\mathrm{av} 2 \max } \geq T_{\mathrm{av} 2 \max 0}, \quad T_{\mathrm{av}} \geq T_{\mathrm{av} 0}, \\
& m \leq m_{0}, \quad L_{\mathrm{wA}} \leq L_{\mathrm{wA} 0}, \quad \varphi_{\mathrm{off}} \leq \varphi_{\mathrm{offmax}},
\end{aligned}
$$



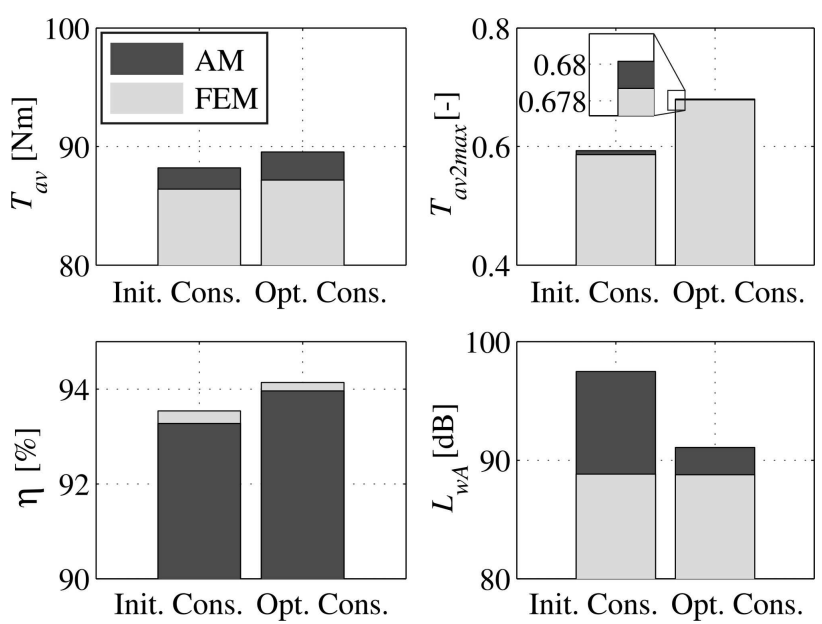

Fig. 8. Values of important motor quantities obtained with the help of an analytical model (AM), and finite element simulations (FEM), for the initial construction and the optimal one - efficiency maximization, problem (18).

Both the above obtained optimal constructions and the initial one have a similar mass of about $46.22 \mathrm{~kg}$.

\section{Conclusions}

The proposed analytical model enables an estimation of the functions necessary in the designing procedure, such as the electromagnetic torque, efficiency and magnetic noise. The model has a feature, which enables its application in an optimization approach with the help of a gradient optimization routine. Analytical solutions are not as accurate as those obtained with the help of FEM, but the calculation time is much lower, and the results are reasonable. It enables a more advanced investigation based on optimization results in the same time. A less accuracy is a reason that results obtained by means of the analytical model should be verified by means of FEM calculations.

\section{Acknowledgement}

The article has been sponsored from the European Union Structural Funds (the ZPORR integrated operational programme in Poland), and from the European Social Fund (ESF), in the project IXC/201/GSI/09.

\section{References}

[1] H. Bausch, A. Greif, B. Lange, and R. Bautz, A 50kW/15000rpm Switched Reluctance Drive for an Electric Vehicle: Current Control and Performance Characteristics. Proceedings of XIV International Conference on Electrical Machines, pp. 603-607, 28-30 August 2000, Espoo, Finland.

[2] Jazdzynski W., Majchrowicz M.:, An Approach to Find an optimum Designed SRM. Proc. of the XVIII Intern. Conf. on Electrical Machines ICEM'08, Paper ID 1391, 6-9 September 2008, Vilamoura, Portugal.

[3] Majchrowicz M., Jazdzynski W.: Selected Aspects of an Optimization of a Switched Reluctance Motor (SRM) for an Electric Vehicle Drive. Zeszyty Problemowe - Maszyny Elektryczne, No. 84 / 2009, pp. $147-150$.

[4] M. Majchrowicz, Analytical Model of Switched Reluctance Motor for its Optimization, Elektrotechnika i Elektronika, Vol. 26, No. 1-2, 2007, pp. 46-53, Cracow, Poland.

[5] W. Jazdzynski, and M. Majchrowicz, Selected Aspects of Switched Reluctance Motor Modeling Based on the Reluctance Network Method, Proceedings of XII Symposium "Fundamental Problems of Power Electronics, Electromechanics and Mechatronics", pp. 123-128, 9-12 December 2007, Wisla, Poland.

[6] Anwar M. N., and I. Husain, Radial Force Calculation and Acoustic Noise Prediction in Switched Reluctance Machines, IEEE Trans. on Industry Applications, Vol. 36, No. 6, 2000, pp. 1589-1597.

[7] A. Matveev, Development of Methods, Algorithms and Software for Optimal Design of Switched Reluctance Drives, Ph.D. thesis, Eindhoven Univ. of Techn., Eindhoven, 2006.

[8] Gieras J. F., Wang C., and Lai J. C., Noise of Polyphase Electric Motors, CRC Press, Taylor \& Francis Group, Boca Raton, 2006. 